\title{
The Orbital Distances Law in Planetary Systems
}

\author{
K. P. Panov*
}

Institute of Astronomy, Bulgarian Academy of Sciences, 72 Tsarigradsko Chausse blvd, 1784 Sofia, Bulgaria

\begin{abstract}
A new approach is proposed for the old problem of the planetary orbital distances in the Solar System. The solution is a simple exponential formula of the type: $a_{n}=C e^{2 n / k}$. The same formula with different parameters could be used also for a large number of satellites of the giant planets Jupiter, Saturn, Uranus, and Neptune. The inner and the most massive satellites follow the respective exponential law, but the less massive outer satellites generally do not. The same exponential law, with different parameters, seems to apply also to the extra-solar planetary systems of 55 Cancri and HD 160691. The general conclusion is that orbital distances in planetary systems are not completely at random: The most massive bodies around stars and planets follow an exponential rule. This is a severe constrain on any theory of the origin of the Solar System. The inclusion of two extra-solar planetary systems (55 Cancri and HD 160691) seems to corroborate this approach also for other planetary systems. The solution for 55 Cancri, however, implies a "missing" planet at $\mathrm{n}=5$. The solution for HD 160691 implies a missing "planet" at $n=2$. The exponential orbital distances law in planetary systems casts serious doubts on existing theories of the origin of the Solar System, which are based on gravitational collapse. Radically new ideas may be necessary to deal with the problem of the origin of planetary systems.
\end{abstract}

\section{INTRODUCTION}

The story of the Titius-Bode law (TBL) for the planetary orbital distances is one of the most interesting in Astronomy. This law has been proposed by the Prussian astronomers J. Titius and J. Bode in 1766, to fit the orbital distances of the planets:

$$
a_{n}=0.4+0.3 \cdot 2^{n}
$$

where $a_{n}$ are the mean distances of the planets from the Sun in astronomical units $\left(1 \mathrm{AU}=149.6 \cdot 10^{6} \mathrm{~km}\right)$, and $\mathrm{n}=-\infty$ for Mercury, $\mathrm{n}=0$ for Venus, $\mathrm{n}=1$ for Earth, and so on. By the time of the TBL publication Saturn $(n=5)$ was the last known planet, since antiquity. It should also be noted that no planet was known at 2.8 AU, i.e. for $n=3$ in the TBL. Bode himself was the first to call attention to this "missing" planet at 2.8 AU, according to the TBL. The community was divided about the meaning of the TBL from the beginning, and many still believe that TBL is a mere coincidence. Yet, for a mere coincidence, the story of the TBL is long and interesting, and obviously goes to the present days. At first, the TBL was quite successful. In $1781 \mathrm{~W}$. Hershel discovered a new planet, Uranus. The distance of Uranus turned out to be 19.2 AU, which corresponds to the predicted by the TBL (for $\mathrm{n}=$ 6) value of 19.6 AU. A major success of the TBL was also the discovery of the minor planet Ceres in 1801, at the predicted by the TBL distance of 2.8 AU. Later on a large number of minor planets - asteroids have been discovered at about the same distance. They are known now as "the asteroid belt". Naturally, the hunt then continued for a planet beyond the orbit of Uranus. The predicted distance for a "transUranus" planet with $\mathrm{n}=7$ was 38.8 AU. Indeed, in $1846 \mathrm{~J}$. Adams and U. LeVerrier discovered Neptune. But the appar-

*Address correspondence to this author at the Institute of Astronomy, Bulgarian Academy of Sciences, 72 Tsarigradsko Chausse blvd, 1784 Sofia, Bulgaria; E-mail: kpanov@astro.bas.bg ent triumph of the TBL stopped: The real distance of Neptune is significantly less than the predicted, i.e. $30.1 \mathrm{AU}$. When the next planet Pluto was discovered in 1930, it became clear that the TBL failed completely. It is presented here only for historical interest.

For TBL -opponents the failure of TBL was the proof that previous success was a coincidence. For others, it meant only that a more accurate formula might exist for the planetary distances, and the TBL was merely an approximation to this unknown formula. The search for such a formula continued to the present day, despite many discouraging statements by leading scientists. (For references see: http://titius-bodelaw.wikiverse.org).

During the last few decades, the progress in space exploration led to the discoveries of a number of new satellites of Jupiter, Saturn, Uranus, and Neptune that resemble the Solar System on a smaller scale. Suggestions have been made for a TBL-type formula for some satellites of the giant planets, but with no conclusive results.

Another exciting development in recent years is the discovery of extra-solar planets. (Presently, their total number is 342 and steadily increasing, see http://exoplanet.eu/). There are five planets discovered in the system of 55 Cancri and four planets in the system of HD 160691.The system of 55 Cancri has been studied in [1], and they proposed a simple exponential formula for the distances of these planets. However, they noted that an "empty orbit" exists in this system for $\mathrm{n}=5$, and attribute it to an undiscovered yet planet. Having in mind the distance to 55 Cancri, new discoveries of planets in this system are not impossible. Poveda and Lara [1] also suggested an exponential formula for the planetary distances in the Solar System, however, they left out Mercury and Pluto from consideration. One gets the impression that the exponential type formula for planetary distances could be a good tool, but some additional improvement of the approach is still necessary. 
In my approach, the solution for the planetary distances should obey to following requirements:

- Generality. The distances law should apply for any system of planets or satellites around a single gravitational center, including the exo-planetary systems.

- Variations of parameters in the formula could be expected and might reflect conditions in different planetary systems.

- In well-known systems, the solution should not create "empty" spaces (orbits), and should apply to all members of the respective system. This later condition may not be fulfilled in exo-planetary systems, if such a system is not yet complete (i.e. not yet all main planets discovered).

It seems possible that an exponential law of the type: $a_{n}=$ C. $\mathrm{e}^{2 \mathrm{n} / \mathrm{k}}$, could fit orbital distances in different systems, where $\mathrm{C}$ and $\mathrm{k}$ are parameters. The above designation of the distances formula is preferable for the following reason. It could be shown that the orbital velocities of the planets in the Solar System $\mathrm{V}_{\mathrm{n}}{ }^{\text {orb }}$ could be fitted by the formula: $\mathrm{V}_{\mathrm{n}}{ }^{\text {orb }}$ $=63.9 \mathrm{e}^{-\mathrm{n} / \mathrm{k}}\left(\mathrm{km} . \mathrm{s}^{-1}\right)$, where $\mathrm{n}=1$ for Mercury, ...., $\mathrm{n}=10$ for Pluto, and $\mathrm{k}=3.722$. Substituting this formula into the $3 \mathrm{rd}$ law of Kepler, we get the expression $a_{n}=C e^{2 n / k}$ where $C$ is different, but $\mathrm{k}$ is the same parameter.

\section{THE SOLAR SYSTEM}

Two approaches for the planetary distances could be considered. First, we could apply a single formula: $\mathrm{km})$,

$a_{n}=31933974 . e^{2 n / 3.722} \quad$ (distances here and further in

where: $n=1-10$, for Mercury, Venus, Earth, Mars, Ceres, Jupiter, Saturn, Uranus, Neptune, and Pluto. With this approach, the largest relative errors $\Delta \mathrm{a} / \mathrm{a}$ are for Mars $(0.20)$, Uranus (0.18), and for Pluto (0.16). Errors could be reduced, if the planetary system is divided in two groups: $n=1-5$ (the inner planets), and $n=6-10$ (the outer planets). With this approach, my suggestion would be:

$$
\begin{aligned}
& \text { - } \quad \text { for } n=1-5, \quad a_{n}=38037460 . \mathrm{e}^{2 \mathrm{n} / 4.28}, \\
& \text { - } \quad \text { and for } \mathrm{n}=6-10, \mathrm{a}_{\mathrm{n}}=37843584 . \mathrm{e}^{2 \mathrm{n} / 3.84} \text {. }
\end{aligned}
$$

Errors in the first group are not greater than 0.10. Errors in the second group are greatest for Pluto (0.17), and Uranus (0.15). The sum of squares of all errors with the second approach is reduced with respect to the first approach by about $40 \%$. Individual errors for planets and satellites could be seen in [2].

For the Jovian system, I shall consider only the first 8 satellites, divided in two groups: $n=1-4$, (Metis, Adrastea, Amaltea, Thebe), and $\mathrm{n}=5-8$ (Io, Europa, Ganymed, Callisto).

$$
\begin{array}{ll}
\text { - For } n=1-4, & a_{n}=97632 \mathrm{e}^{2 \mathrm{n} / 10.05}, \text { and } \\
\text { - for } \mathrm{n}=5-8, & \mathrm{a}_{\mathrm{n}}=34704 \mathrm{e}^{2 \mathrm{n} / 4.04} .
\end{array}
$$

The largest error is for Adrastea (0.13). It should be noted that all the other satellites of Jupiter with $n>8$ have a little contribution to the total mass of the Jovian system of satellites.
For the system of Saturn I will consider the first 22 satellites, divided into 4 groups: $\mathrm{n}=1-7$ (Pan, S/2005 S1, Atlas, Prometheus, Pandora, Epimetheus, Janus), n $=8-14$ (Mimas, S/2004 S1, S/2004 S2, Enceladus, Tethis, Dione, Rhea), $\mathrm{n}=15-17$ (Titan, Hyperion, Iapetus), and $\mathrm{n}=18-$ 22 (Kiviuq, Ljirak, Phoebe, Paaliak, Skathi). The respective formulas are:

$$
\begin{aligned}
\text { - } & \text { for } \mathrm{n}=1-7, \quad \mathrm{a}_{\mathrm{n}}=129679 . \mathrm{e}^{2 \mathrm{n} / 91.42} \\
\text { - } & \text { for } \mathrm{n}=8-14, \quad \mathrm{a}_{\mathrm{n}}=41103 . \mathrm{e}^{2 \mathrm{n} / 11.68} \\
\text { - } & \text { for } \mathrm{n}=15-17, \quad \mathrm{a}_{\mathrm{n}}= \\
\text { - } & \text { for } \mathrm{n}=18-22, \mathrm{e}^{2 \mathrm{n} / 3.74} \\
\text { a } & =2080415 . \mathrm{e}^{2 \mathrm{n} / 21.65}
\end{aligned} \text {. }
$$

Remarkably, $\mathrm{k}$ is decreasing from group 1 to group 3 , but increases again in the last group 4.

For Uranus, it is possible to study all 27 known satellites, and also the rings, because of the sharp distances of the rings from the planet. All 11 rings could be fitted by: $a_{n}=38657$. $\mathrm{e}^{2 \mathrm{n} / 76.41}$, where $\mathrm{n}=1-11(1986 \mathrm{U} 2 \mathrm{R}, 6,5,4$, Alpha, Beta, Eta, Gamma, Delta, 1986 U1R, Epsilon). The 27 satellites are divided into 4 groups, as follows:

$\mathrm{n}=1-6$ (Cordelia, Ophelia, Bianca, Cressida, Desdemona, Juliet), $\mathrm{n}=7-13$ (Portia, Rosalind, S/ 2003 U2, Belinda, S/ 1986 U10, Puck, S/2003 U1), n = 14 - 18 (Miranda, Ariel, Umbriel, Titania, Oberon), and $n=19-27$ (S/2001U3, Caliban, Stephano, Trinkulo, Sycorax, S/2003U3, Prospero, Setebos, $\mathrm{S} / 2001 \mathrm{U} 2)$. The respective formuas are:

$$
\begin{aligned}
& \text { - } \text { for } n=1-6, \quad a_{n}=48821 . e^{2 n / 39.16} \\
& \text { - } \text { for } n=7-13, a_{n}=43593 . e^{2 n / 34.8} \\
& \text { - for } n=14-18, a_{n}=607 . e^{2 n / 5.22} \\
& \text { - } \quad \text { for } n=19-27, a_{n}=165511 . e^{2 n / 10.985} \text {. }
\end{aligned}
$$

The relative errors for the rings and for the first 3 groups of satellites of Uranus are tolerable - not greater than 0.06.In the last (outermost) group of satellites, errors increase somewhat. This is not a surprise, since the outermost small mass satellites are most prone to gravitational perturbations. Again, we can see that the $\mathrm{k}$ is decreasing with increasing distance from the planet, but $\mathrm{k}$ increases again in the most distant group of satellites.

For the system of Neptune, only the first 7 satellites are considered, divided in 2 groups. The first group is: $n=1-4$, (Naiad, Thalassa, Despina, Galatea), and the second group is $n=5-7$ (Larissa, Proteus, Triton). The respective formulas are:

$$
\begin{aligned}
& \text { - } \text { for } n=1-4, \quad a_{n}=43328 . e^{2 n / 24.93} \\
& \text { - } \text { for } n=5-7, \quad a_{n}=1292 . e^{2 n / 2.54}
\end{aligned}
$$
0.24 .

In the system of Neptune, the largest error is for Proteus,

\section{COMMENTS}

All solutions presented are least squares solutions. Two remarks deserve attention. In this study, I have included the retrograde satellites Phoebe (Saturn) and Triton (Neptune). These are included in groups of other, ordinary (direct) satellites. Since the respective formulas seem to work also for the retrograde satellites, their origin could not be so much different from the origin of the ordinary satellites. In all systems 
studied, the $\mathrm{k}$ parameter decreases first with increasing distance from the respective planet. This is also the case for the Solar System, if we consider the inner and the outer planets separately. In the systems of Saturn and Uranus, however, where the study is possible also to more distant groups of satellites, $\mathrm{k}$ seems to increase again. This is an interesting trend, but unfortunately, no physical implications are possible yet. Errors are usually tolerable. Only in a few cases they are unusually large: 0.23 for S/2001U3, (Uranus), 0.24 for Proteus (Neptune), and 0.26 for Hyperion (Saturn). It should be noted that these are small mass satellites and the errors could be due to gravitational perturbation and dynamical evolution. In all systems, application of the respective formula starts with $\mathrm{n}=1$ and proceeds to some outer satellite. The non-included outer satellites represent only a minor contribution to the total mass of the respective system of satellites considered. There are cases where at about the same distance from the planet more than one satellite exist, e.g. in the system of Saturn, Thetis, Telesto, and Calypso are each about $294700 \mathrm{~km}$ away from this planet. In such cases, the same orbital number refers to all of these satellites. The general conclusion is that exponential law of orbital distances in the Solar System exists and should be accounted for by any theory, pretending to reveal the origin of the Solar System. Let us now turn to the exo-planetary systems of 55 Cancri and HD 160691 and apply the same approach.

\section{THE EXO-PLANETARY SYSTEMS OF 55 CANCRI AND HD 160691.}

Only in two exo-planetary systems there are several planets each and we could study the orbital distances with reasonable number of planets. It is clear that these exoplanetary systems are probably not yet complete and new discoveries of planets are likely. The system of 55 Cancri (five planets) has already been studied in [1]. I will therefore suggest the distances formula: $a_{n}=2096572 . e^{2 n / 2.002}$. Details are presented in Table 1. Distances of the planets from the star are in $\mathrm{km}$.

Table 1. Planetary System of 55 Cancri

\begin{tabular}{|c|c|c|}
\hline Orbital Number, $\mathbf{n}$ & $\begin{array}{c}\text { Distance from the } \\
\text { Star, Km }\end{array}$ & Relative Error \\
\hline \hline $\mathrm{n}=1$ & $5.6848 .10^{6}$ & 0.00 \\
\hline $\mathrm{n}=2$ & $17.2040 .10^{6}$ & -0.10 \\
\hline $\mathrm{n}=3$ & $35.9040 .10^{6}$ & 0.17 \\
\hline $\mathrm{n}=4$ & $116.8376 .10^{6}$ & -0.02 \\
\hline $\mathrm{n}=5$ & Missing & \\
\hline $\mathrm{n}=6$ & $863.1920 .10^{6}$ & -0.03 \\
\hline
\end{tabular}

The formula seems to work and errors are tolerable, comparable to the errors in the Solar System study. However, there is a "missing" planet with $\mathrm{n}=5$, i.e. at about 2.1 AU from the star. This result is similar to the conclusion of Poveda and Lara.

Only four planets have been discovered in the system of HD 160691. The formula: $\mathrm{a}_{\mathrm{n}}=5945569 . \mathrm{e}^{2 \mathrm{n} / 2.123}$ seems to fit the orbital distances, and the details are shown in Table $\mathbf{2}$.
Table 2. Planetary System of HD 160691

\begin{tabular}{|c|c|c|}
\hline Orbital Number, $\mathbf{n}$ & $\begin{array}{c}\text { Distance from the } \\
\text { Star, } \mathbf{k m}\end{array}$ & Relative Error \\
\hline \hline $\mathrm{n}=1$ & $13.464 .10^{6}$ & 0.13 \\
\hline $\mathrm{n}=2$ & Missing & \\
\hline $\mathrm{n}=3$ & $137.782 .10^{6}$ & -0.27 \\
\hline $\mathrm{n}=4$ & $224.400 .10^{6}$ & 0.15 \\
\hline $\mathrm{n}=5$ & $623.832 .10^{6}$ & 0.06 \\
\hline
\end{tabular}

Apparently, in the system of HD 160691 there is a "missing" planet for $n=2$. The respective distance from the star is about $0.26 \mathrm{AU}$. The largest error is for planet $\mathrm{n}=3,-0.27$ (at $0.92 \mathrm{AU}$ from the star). The missing planets at such great distances from the observer are not a surprise, nor are the large errors. Future studies will show whether or not these missing planets exist.

\section{CONCLUSIONS}

In all systems studied, exponential formulas of the type $\mathrm{a}_{\mathrm{n}}$ $=\mathrm{C} \cdot \mathrm{e}^{2 \mathrm{n} / \mathrm{k}}$ have been applied, with different parameters $\mathrm{C}$ and $\mathrm{k}$ in each case. All values of the parameters are summarized in Table 3. All parameters are least squares solutions.

From this study, following conclusions seem appropriate:

- There is substantial evidence against a random, chaotic distribution of orbits of planets and their satellites. Orbital distances seem to obey a simple exponential law which probably reflects real conditions during the building of the respective system.

- Remarkably, for different systems different parameters in the exponential formula should be applied. It could be an indication that one and the same process was repeated during the building of different planetary systems and systems of satellites, but under different conditions. This is probably the reason why so many different TBL-type solutions have been proposed in the past.

- The exponential distances law seems to work also in two exo-planetary systems, 55 Cancri and HD 160691. It may be a hint that the same distances law could be applied to other planetary systems. Remarkably, the values of $\mathrm{k}$ for 55 Cancri (2.002) and for HD 160691 (2.123) are not much different from the value of k of the Solar System.

- In all systems of satellites studied in the Solar System, the value of $k$ decreases first with increasing distance from the gravitational center. In the outermost groups of satellites of Saturn and Uranus, the value of $\mathrm{k}$ increases again with the distance from the respective planet. An important difference between the Solar System (main planets) and the systems of satellites of the giant planets is the following. In the Solar System, with increasing distance from the Sun parameters $\mathrm{C}$ and $\mathrm{k}$ do not change very much. This makes it possible to introduce a single set of parameters, $\mathrm{C}=31933974$, and $\mathrm{k}=$ 
Table 3. Summary of Parameters of Solutions. Mean errors are Given in Brackets

\begin{tabular}{|c|c|c|c|}
\hline System & $\begin{array}{c}\text { Orbital } \\
\text { Number, } \mathbf{n}\end{array}$ & C & $\mathbf{k}$ \\
\hline Solar system & $\mathrm{n}=1-5$ & $38037460\left(+3.6 .10^{6},-3.3 .10^{6}\right)$ & $4.28 \quad(-0.18,+0.19)$ \\
\hline Solar system & $\mathrm{n}=6-10$ & $37843584\left(+18 \cdot 10^{6},-12 \cdot 10^{6}\right)$ & $3.84(-0.3,+0.3)$ \\
\hline 55 Cancri & $n=1-6$ & $2096572\left(+0.2 .10^{6},-0.2 .10^{6}\right)$ & $2.002(-0.04,+0.04)$ \\
\hline Jupiter & $\mathrm{n}=1-4$ & $97632\left(+1.0 .10^{4},-0.9 .10^{4}\right)$ & $10.05(-1.7,+2.5)$ \\
\hline Jupiter & $\mathrm{n}=5-8$ & $34704\left(+3.6 \cdot 10^{3},-6.9 .10^{3}\right)$ & $4.04 \quad(-0.1,+0.1)$ \\
\hline Saturn & $\mathrm{n}=1-7$ & $129679\left(+1.3 \cdot 10^{3},-1.3 \cdot 10^{3}\right)$ & $91.42(-14,+20)$ \\
\hline Saturn & $\mathrm{n}=8-14$ & $41103\left(+9.1 \cdot 10^{3},-7.5 \cdot 10^{3}\right)$ & $11.68(-1.2,+1.5)$ \\
\hline Uranus & Rings & $38657(+388,-385)$ & $76.41(-4.9,+5.6)$ \\
\hline Uranus & $\mathrm{n}=1-6$ & $48821 \quad\left(+1.5 \cdot 10^{3},-1.4 \cdot 10^{3}\right)$ & $39.16(-5.3,+7.3)$ \\
\hline Uranus & $\mathrm{n}=7-13$ & $43593\left(+4.1 \cdot 10^{3},-3.7 \cdot 10^{3}\right)$ & $34.80(-4.3,+5.6)$ \\
\hline Uranus & $\mathrm{n}=14-18$ & $607(+180,-139)$ & $5.22 \quad(-0.2,+0.2)$ \\
\hline Uranus & $\mathrm{n}=19-27$ & $165511\left(+5.8 \cdot 10^{4},-4.3 \cdot 10^{4}\right)$ & $10.985(-0.9,+1.1)$ \\
\hline Neptune & $\mathrm{n}=1-4$ & $43328\left(+2.7 \cdot 10^{3},-2.5 \cdot 10^{3}\right)$ & $24.93(-5,+8)$ \\
\hline Neptune & $\mathrm{n}=5-7$ & $1292\left(+2.6 \cdot 10^{3},-0.9 \cdot 10^{3}\right)$ & $2.54 \quad(-0.5,+0.7)$ \\
\hline
\end{tabular}

3.722 , to fit distances of all main planets $(n=1$ for Mercury,......, $n=10$ for Pluto). The situation in all systems of satellites studied is different. The changes of the parameters in each system of satellites are considerable and because of that a single set of parameters could not be established, which would fit the distances of all satellites in the system. It is not clear, what is the reason for that different behaviour, as we do not yet know the physics behind the exponential distances rule. Because of the strong changes in the parameters in each system of satellites, sometimes groups of 3-4 satellites have to be considered, in order to produce a reasonable distances fit. On the other hand, for such small samples of satellites, statistical (mean) errors of the parameters (see Table 3) are quite considerable.

- The exponential distances law probably reflects physical conditions of the origin of planetary systems. Any theory, pretending to describe the origin of planetary systems should be able to deal with the distances law.

- The exponential distances law seems to contradict present theories of the origin of the Solar System, based on gravitational collapse. There is another obstacle for these theories, in my opinion. In many exo-planetary systems large planets have been discovered very close to the respective star. In the system of 55 Cancri, there are planets at $0.04 \mathrm{AU}$ and $0.12 \mathrm{AU}$, respectively. In HD 160691, there is a planet at 0.09 AU. How was it possible for the gravitational collapse to succeed in building planets so close to the respective star? There are suggestions that planets have originally been built somewhere far away from the star and then managed to spiral down to the star by dynamical evolution. They now appear near the respective star. Inward migration of planetary cores through interactions with the remnants of the circumstellar disk [3] , or migration through gas drag [4] have been invoked to deal with this problem. Having in mind the possibility of a distances law for exo-planetary systems, it would be highly unlikely that dynamical evolution could bring planets to "end-positions", which obey the respective distances law. An alternative theory is not in view, however, radically new ideas may be necessary to explain the origin of the planetary systems and the orbital distances law.

\section{ACKNOWLEDGEMENTS}

I am grateful to Prof Oliver Manuel, for critically reviewing the manuscript.

\section{REFERENCES}

[1] Poveda A, Lara P. The exo-planetary system of 55 cancri and the titius-Bode law. Revista Mexicana de Astronomia y Astrofisica 2008; 44: 243-6.

[2] Panov K. The law of orbital distances in the Solar system. Comptes Rendus Acad. Bulgare des Sciences 2009; 62(2): 143-52. 
[3] Lin DNC, Bodenheimer P, Richardson DC. Orbital migration of the planetary companion of 51 Pegasi to its present location. Nature 1996; 380(6575): 606-7.
[4] Kary DM, Lissauer JJ. Nebular gas drag and planetary accretion.II. Planet on an eccentric orbit. Icarus 1995; 117(09): 1-24

Received: April 23, 2009

Revised: August 25, 2009

Accepted: August 30, 2009

(c) K. P. Panov; Licensee Bentham Open.

This is an open access article licensed under the terms of the Creative Commons Attribution Non-Commercial License (http://creativecommons.org/licenses/by-nc/3.0/) which permits unrestricted, non-commercial use, distribution and reproduction in any medium, provided the work is properly cited. 